\title{
AN OPERATOR-VALUED YEH-WIENER INTEGRAL AND A KAC-FEYNMAN WIENER INTEGRAL EQUATION
}

\author{
CHULL PARK AND DAVID SKOUG
}

(Communicated by Lawrence F. Gray)

\begin{abstract}
Let $C[0, T]$ denote Wiener space, i.e., the space of all continuous functions $\eta(t)$ on $[0, T]$ such that $\eta(0)=0$. For $Q=[0, S] \times[0, T]$ let $C(Q)$ denote Yeh-Wiener space, i.e., the space of all $\mathbb{R}$-valued continuous functions $x(s, t)$ on $Q$ such that $x(0, t)=x(s, 0)=0$ for all $(s, t)$ in $Q$. For $h \in L_{2}(Q)$ let $Z(x ; s, t)$ be the Gaussian process defined by the stochastic integral$$
Z(x ; s, t)=\int_{0}^{t} \int_{0}^{s} h(u, v) d x(u, v)
$$

Then for appropriate functionals $F$ and $\psi$ we show that the operator-valued function space integral

$$
\left(I_{\lambda}^{h}(F) \psi\right)(\eta(\cdot))=E_{x}\left[F\left(\lambda^{-1 / 2} Z(x ; \cdot, \cdot)+\eta(\cdot)\right) \psi\left(\lambda^{-1 / 2} Z(x ; S, \cdot)+\eta(\cdot)\right)\right]
$$

is the unique solution of a Kac-Feynman Wiener integral equation. We also use this integral equation to evaluate various Yeh-Wiener integrals.
\end{abstract}

\section{INTRODUCTION}

For $Q=[0, S] \times[0, T]$ let $C(Q)$ denote Yeh-Wiener space. Yeh [12] defined a Gaussian measure $m_{y}$ on $C(Q)$ (later modified in [13]) such that as a stochastic process $\{x(s, t):(s, t) \in Q\}$ has mean $E[x(s, t)]=\int_{C(Q)} x(s, t) m_{y}(d x)=$ 0 and covariance $E[x(s, t) x(u, v)]=\min \{s, u\} \min \{t, v\}$. Let $C_{w} \equiv C[0, T]$ denote the standard Wiener space on $[0, T]$ with Wiener measure $m_{w}$. For $0 \leq s<S$, let $Q_{s}=[s, S] \times[0, T]$. The corresponding Yeh-Wiener space is denoted by $C\left(Q_{s}\right)$ and we use $m_{y}$ for the Yeh-Wiener measure on $C(Q)$ as well as on $C\left(Q_{s}\right)$. The same convention holds in the one time-parameter case. We also need $C^{*}(Q)$, the space of $\mathbb{R}$-valued continuous functions $x(s, t)$ on $Q$ such that $x(\cdot, 0)=0$.

Consider the operator-valued integral in function space $I_{\lambda}(F) \equiv I_{\lambda,[0, T]}(F)$

Received by the editors June 18, 1992.

1991 Mathematics Subject Classification. Primary 60J65, 28C20, $60 \mathrm{G} 15$.

Key words and phrases. Yeh-Wiener itegral, Wiener integral equation, Gaussian process.

(c) 1994 American Mathematical Society 
which takes the function $\psi$ into the function $i_{\lambda}(F) \psi$ whose value at $\xi \in \mathbb{R}$ is

$$
\begin{aligned}
\left(I_{\lambda}(F) \psi\right)(\xi) & \equiv \int_{C[0, T]} F\left(\lambda^{-1 / 2} x+\xi\right) \psi\left(\lambda^{-1 / 2} x(T)+\xi\right) m_{w}(d x) \\
& \equiv E_{x}\left[F\left(\lambda^{-1 / 2} x+\xi\right) \psi\left(\lambda^{-1 / 2} x(T)+\xi\right)\right] \quad(\lambda>0) .
\end{aligned}
$$

A very important class of functions in quantum mechanics consists of functions on $C[0, T]$ of the type

$$
F(x)=\exp \left\{\int_{0}^{T} \theta(s, x(s)) d s\right\},
$$

where $\theta:[0, T] \times \mathbb{R} \rightarrow \mathbb{C}$.

In fact, it is well known that if $\theta(s, u)$ is bounded and continuous on $[0, T] \times \mathbb{R}$, then the operator $I_{\lambda}(F)$ transforms $L_{2}[0, T]$ into $L_{2}[0, T]$. Furthermore, if we define

$$
F_{t}(x) \equiv \exp \left\{\int_{t}^{T} \theta(s, x(s)) d s\right\} \quad(0 \leq t<T),
$$

then the function

$$
G(t, \xi, \lambda)=\left(I_{\lambda,[t, T]}\left(F_{t}\right) \psi\right)(\xi)
$$

satisfies the Kac-Feynman integral equation

$$
\begin{aligned}
G(t, \xi, \lambda) & \\
= & {\left[\frac{\lambda}{2 \pi(T-t)}\right]^{1 / 2} \int_{\mathbb{R}} \psi(v) \exp \left\{\frac{-\lambda(v-\xi)^{2}}{2(T-t)}\right\} d v } \\
& +\left(\frac{\lambda}{2 \pi}\right)^{1 / 2} \int_{t}^{T}(s-t)^{-1 / 2} \int_{\mathbb{R}} \theta(s, v) G(s, v, \lambda) \exp \left\{\frac{-\lambda(v-\xi)^{2}}{2(s-t)}\right\} d v d s .
\end{aligned}
$$

If $\theta$ is sufficiently smooth, then this equation implies the backwards diffusion equation

$$
\frac{1}{2 \lambda} \frac{\partial^{2} G}{\partial \xi^{2}}+\frac{\partial G}{\partial t}+\theta G=0
$$

with the boundary condition

$$
\lim _{t \rightarrow T^{-}} G(t, \xi, \lambda)=\psi(\xi) .
$$

The celebrated paper [4] by Cameron and Storvick considered the operatorvalued function space integral $I_{\lambda}(F) \equiv I_{\lambda, Q}(F)$ which maps the functional $\psi$ into the functional $I_{\lambda}(F) \psi$ whose value at $\eta(\cdot) \in C[0, T]$ is given by

$$
\begin{aligned}
\left(I_{\lambda}(F) \psi\right)(\eta(\cdot)) & \equiv \int_{C(Q)} F\left(\lambda^{-1 / 2} x(\cdot, \cdot)+\eta(\cdot)\right) \psi\left(\lambda^{-1 / 2} x(S, \cdot)+\eta(\cdot)\right) m_{y}(d x) \\
& \equiv E_{x}\left[F\left(\lambda^{-1 / 2} x(\cdot, \cdot)+\eta(\cdot)\right) \psi\left(\lambda^{-1 / 2} x(S, \cdot)+\eta(\cdot)\right)\right] \quad(\lambda>0) .
\end{aligned}
$$

In particular, if

$$
F_{s}(x(\cdot, \cdot)) \equiv \exp \left\{\int_{s}^{S} \theta(\sigma, x(\sigma, \cdot)) d \sigma\right\},
$$


then

$$
\begin{aligned}
\left(I_{\lambda, Q_{s}}\left(F_{s}\right) \psi\right)(\eta(\cdot)) & \\
& \equiv \int_{C\left(Q_{s}\right)} F_{s}\left(\lambda^{-1 / 2} x(\cdot, \cdot)+\eta(\cdot)\right) \psi\left(\lambda^{-1 / 2} x(S, \cdot)+\eta(\cdot)\right) m_{y}(d x) \\
& \equiv E_{x}\left[F_{s}\left(\lambda^{-1 / 2} x(\cdot, \cdot)+\eta(\cdot)\right) \psi\left(\lambda^{-1 / 2} x(S, \cdot)+\eta(\cdot)\right) \mid x(s, \cdot)=0\right] .
\end{aligned}
$$

One of the main results of Cameron and Storvick in [4] can be stated as follows:

Theorem A (Cameron and Storvick). Let $\theta(\sigma, x(\sigma, \cdot))$ be bounded and continuous on $[0, S] \times[0, T]$, and let $\psi(\gamma w(\cdot)+\eta(\cdot))$ be Wiener integrable in $w$ for each $\gamma>0$ and each $\eta \in C[0, T]$. For $\lambda>0$ and $\rho=\lambda^{-1 / 2}$, let $F_{s}(x)$ and $G(s, \eta(\cdot)) \equiv\left(I_{\lambda, Q_{s}}\left(F_{s}\right) \psi\right)(\eta(\cdot))$ be defined by (1.3) and (1.4), respectively. Then $G(s, \eta(\cdot))$ satisfies the Kac-Feynman Wiener integral equation

$$
\begin{aligned}
G(s, \eta(\cdot))= & E_{w}[\psi(\rho \sqrt{S-s} w(\cdot)+\eta(\cdot))] \\
& +\int_{s}^{s} E_{w}[\theta(\sigma, \rho \sqrt{\sigma-s} w(\cdot)+\eta(\cdot)) G(\sigma, \rho \sqrt{\sigma-s} w(\cdot)+\eta(\cdot))] d \sigma .
\end{aligned}
$$

Remark. Equation (1.5) is slightly different from the equation given by Cameron and Storvick. The reason is that while they used the original "old" Yeh-Wiener process with covariance $E[x(s, t) x(u, v)]=\frac{1}{2} \min \{s, u\} \min \{t, v\}$, we used the standard Yeh-Wiener process given earlier in the introduction.

In this paper we generalize and extend considerably the results in [4]. In particular we replace the Yeh-Wiener process $x(s, t)$ with the Gaussian process $Z(x ; s, t)$. Moreover our proofs are much simpler because we employ probabilistic techniques extensively.

\section{THE OPERATOR-VALUED INTEGRAL $I_{\lambda}^{h}(F)$}

For each $h \in L_{2}(Q)$ and $\lambda>0$, consider the operator-valued integral $I_{\lambda}^{h}(F) \equiv I_{\lambda, Q}^{h}(F)$ which transforms the functional $\psi$ into the functional $I_{\lambda}^{h}(F) \psi$ whose value at $\eta(\cdot) \in C[0, T]$ is given by

$$
\begin{aligned}
&\left(I_{\lambda}^{h}(F) \psi\right)(\eta(\cdot)) \\
& \equiv \int_{C(Q)} F\left(\lambda^{-1 / 2} \int_{0}^{\cdot} \int_{0}^{\cdot} h(u, v) d x(u, v)+\eta(\cdot)\right) \\
& \cdot \psi\left(\lambda^{-1 / 2} \int_{0}^{\cdot} \int_{0}^{S} h(u, v) d x(u, v)+\eta(\cdot)\right) m_{y}(d x) \\
& \equiv E_{x}\left[F\left(\lambda^{-1 / 2} \int_{0}^{\cdot} \int_{0}^{\cdot} h(u, v) d x(u, v)+\eta(\cdot)\right)\right. \\
&\left.\cdot \psi\left(\lambda^{-1 / 2} \int_{0}^{\cdot} \int_{0}^{s} h(u, v) d x(u, v)+\eta(\cdot)\right)\right] .
\end{aligned}
$$


Since $\int_{0}^{*} \int_{0}^{*} 1 d x(u, v)=x(\cdot, *)$, the Cameron-Storvick operator-valued integral $I_{\lambda}(F)$ given by (1.2) is a special case of $I_{\lambda}^{h}(F)$ with $h \equiv 1$ on $Q$, i.e., $I_{\lambda}(F)=I_{\lambda}^{1}(F)$. For notational simplicity, we define

$Z(x ; s, t) \equiv Z_{h}(x ; s, t)=\int_{0}^{t} \int_{0}^{s} h(u, v) d x(u, v), \quad x \in C(Q),(s, t) \in Q$

Thus, $Z(x ; \cdot, \cdot)$ is a stochastic integral which is a Gaussian process with mean 0 and covariance

$$
E\left[Z(x ; s, t) Z\left(x ; s^{\prime}, t^{\prime}\right)\right]=\int_{0}^{t^{*} t^{\prime}} \int_{0}^{s^{\hat{s}^{\prime}} s^{\prime}} h^{2}(u, v) d u d v
$$

where $t^{\wedge} t^{\prime}=\min \left\{t, t^{\prime}\right\}$. Since the covariance function of $Z(x ; \cdot, \cdot)$ is continuous, we may assume that almost every sample path of $Z(x ; \cdot, \cdot)$ is in $C(Q)$ [7, p. 157]. Obviously $Z(x ; \cdot, \cdot)$ has independent increments, and in terms of $Z,(2.1)$ becomes

$$
\left(I_{\lambda}^{h}(F) \psi\right)(\eta(\cdot))=E_{x}\left[F\left(\lambda^{-1 / 2} Z(x ; \cdot, \cdot)+\eta(\cdot)\right) \psi\left(\lambda^{-1 / 2} Z(x ; S, \cdot)+\eta(\cdot)\right)\right]
$$

Let $\mathscr{A}(C[0, T])$ be the class of functionals $\psi$ on $C[0, T]$ such that $\psi\left(\int_{0}^{*} k(u) d w(u)+\eta(\cdot)\right)$ is Wiener integrable in $w$ over $C[0, T]$ for every $k \in L_{2}[0, T]$ and $\eta \in C[0, T]$. Our first objective is to show that under suitable assumptions on $F$, and for $\lambda>0$,

$$
I_{\lambda}^{h}(F): \mathscr{A}(C[0, T]) \rightarrow \mathscr{A}(C[0, T]) .
$$

As usual, two Gaussian processes are said to be equivalent if they have identical mean and covariance functions. We start with the following lemma.

Lemma 1. Let $h \in L_{2}(Q)$ and $k \in L_{2}[0, T]$. Let $x$ be the Yeh-Wiener process on $Q$, and let $w$ be the standard Wiener process on $[0, T]$. If $x$ and $w$ are independent, then for each $\rho>0$, the two processes $\rho Z(x ; S, \cdot)+\int_{0}^{*} k(u) d w(u)$ and $\int_{0}^{0} p(v) d w_{1}(v)$ are equivalent, where $w_{1}$ is the standard Wiener process on $[0, T]$ and $p(v)=\left[\rho^{2} \int_{0}^{S} h^{2}(u, v) d u+k^{2}(v)\right]^{1 / 2}$.

Proof. The two processes are Gaussian processes on $[0, T]$ with mean zero and identical covariance. Thus, they are equivalent Gaussian processes.

Theorem 1. Let $F$ be a bounded functional, continuous in the uniform topology on $C^{*}(Q)$. Then for each $\lambda>0$ and $h \in L_{2}(Q), I_{\lambda}^{h}(F)$ maps $\mathscr{A}(C[0, T])$ into $\mathscr{A}(C[0, T])$.

Proof. Let $\psi \in \mathscr{A}(C[0, T])$ and $|F| \leq M$. We need to show that $\left(I_{\lambda}^{h}(F) \psi\right) \cdot$ $\left(\int_{0}^{\bullet} k(u) d w(u)+\eta(\cdot)\right)$ is Wiener integrable in $w$ over $C[0, T]$ for every $k \epsilon$ 
$L_{2}[0, T]$ and $\eta \in C[0, T]$. Now, with $\rho=\lambda^{-1 / 2}$,

$$
\begin{gathered}
E_{w}\left[\left|\left(I_{\lambda}^{h}(F) \psi\right)\left(\int_{0}^{\cdot} k(u) d w(u)+\eta(\cdot)\right)\right|\right] \\
\leq E_{w}\left(E _ { x } \left[\mid F\left(\rho Z(x ; \cdot, \cdot)+\int_{0}^{\cdot} k(u) d w(u)+\eta(\cdot)\right)\right.\right. \\
\left.\left.\cdot \psi\left(\rho Z(x ; S, \cdot)+\int_{0}^{\cdot} k(u) d w(u)+\eta(\cdot)\right) \mid\right]\right) \\
\leq M E_{w}\left(E_{x}\left[\left|\psi\left(\rho Z(x ; S, \cdot)+\int_{0}^{0} k(u) d w(u)+\eta(\cdot)\right)\right|\right]\right) \\
=M E_{w_{1}}\left[\left|\psi\left(\int_{0}^{\cdot}\left(\rho^{2} \int_{0}^{S} h^{2}(u, v) d u+k^{2}(v)\right)^{1 / 2} d w_{1}(v)+\eta(\cdot)\right)\right|\right],
\end{gathered}
$$

where the last equality follows from Lemma 1 . Since $\psi \in \mathscr{A}(C[0, T])$, the last expression is finite, and hence the proof is complete.

Next we compute $I_{\lambda}^{h}(F) \psi$ for a given function $F$ which is continuous but is not bounded.

Example 1. Let $\left(S_{1}, T_{1}\right) \in(0, S) \times(0, T]$ and let $F(x)=\exp \left\{x\left(S_{1}, T_{1}\right)\right\}$. Then for $\psi \in \mathscr{A}(C[0, T]), h \in L_{2}(Q)$, and $\lambda>0$ we obtain that

$$
\begin{aligned}
&\left(I_{\lambda}^{h}(F) \psi\right)(\eta(\cdot))= \int_{C(Q)} \exp \left\{\lambda^{-1 / 2} \int_{0}^{T_{1}} \int_{0}^{S_{1}} h d x+\eta\left(T_{1}\right)\right\} \\
& \cdot \psi\left(\lambda^{-1 / 2} \int_{0}^{T_{1}} \int_{0}^{S} h d x+\eta\left(T_{1}\right)\right) m_{y}(d x) \\
&=(2 \pi)^{-1} \int_{\mathbf{R}^{2}} \exp \left\{\lambda^{-1 / 2}\left(\int_{0}^{T_{1}} \int_{0}^{S_{1}} h^{2}\right)^{1 / 2} u_{1}+\eta\left(T_{1}\right)-\frac{u_{1}^{2}}{2}-\frac{u_{2}^{2}}{2}\right\} \\
& \cdot \psi\left(\lambda^{-1 / 2}\left(\int_{0}^{T_{1}} \int_{0}^{S_{1}} h^{2}\right)^{1 / 2} u_{1}\right. \\
&\left.\quad+\lambda^{-1 / 2}\left(\int_{0}^{T_{1}} \int_{S_{1}}^{S} h^{2}\right)^{1 / 2} u_{2}+\eta\left(T_{1}\right)\right) d u_{2} d u_{1} \\
&=\left(\frac{\lambda}{2 \pi \int_{0}^{T_{1}} \int_{0}^{S_{1}} h^{2}}\right)^{1 / 2}\left(\frac{\lambda}{2 \pi \int_{0}^{T_{1}} \int_{S_{1}}^{S} h^{2}}\right)^{1 / 2} \\
& \cdot \int_{\mathbf{R}^{2}} \psi\left(v_{2}\right) \exp \left\{v_{1}-\frac{\lambda\left(v_{1}-\eta\left(T_{1}\right)\right)^{2}}{2 \int_{0}^{T_{1}} \int_{0}^{S_{1}} h^{2}}-\frac{\lambda\left(v_{2}-v_{1}\right)^{2}}{2 \int_{0}^{T_{1}} \int_{S_{1}}^{S} h^{2}}\right\} d v_{2} d v_{1} .
\end{aligned}
$$


Example 2. Choosing $F(x)=\exp \left\{x\left(S, T_{1}\right)\right\}$ and proceeding as above we obtain that

$$
\left(I_{\lambda}^{h}(F) \psi\right)(\eta(\cdot))=\left(\frac{\lambda}{2 \pi \int_{0}^{T_{1}} \int_{0}^{S} h^{2}}\right)^{1 / 2} \int_{\mathbb{R}} \psi(v) \exp \left\{v-\frac{\lambda\left(v-\eta\left(T_{1}\right)\right)^{2}}{2 \int_{0}^{T_{1}} \int_{0}^{S} h^{2}}\right\} d v
$$

3. THE OPERATOR $I_{\lambda, Q_{s}}^{h}\left(F_{s}\right)$ AND AN INTEGRAL EQUATION

Let $Z(x ; s, t)$ be as before, and let $F_{s}$ be given by (1.3) so that

$$
F_{s}(Z(x)) \equiv F_{s}(Z(x ; *, \cdot))=\exp \left\{\int_{s}^{s} \theta(\sigma, Z(x ; \sigma, \cdot)) d \sigma\right\}
$$

$(0 \leq s<S)$. Define the operator $I_{\lambda, Q_{s}}^{h}\left(F_{s}\right)$ with $\rho=\lambda^{-1 / 2}, \lambda>0$, by

$$
\begin{aligned}
& \left(I_{\lambda, Q_{s}}^{h}\left(F_{s}\right) \psi\right)(\eta(\cdot)) \\
& \quad \equiv E\left[F_{s}(\rho Z(x ; *, \cdot)+\eta(\cdot)) \psi(\rho Z(x ; S, \cdot)+\eta(\cdot)) \mid Z(x ; s, \cdot)=0\right] .
\end{aligned}
$$

We start with the following lemma whose proof is immediate by checking the mean and covariance functions.

Lemma 2. Let $h \in L_{2}(Q)$ and let $x$ be a Yeh-Wiener process on $Q$. Let $0 \leq s \leq \sigma \leq S$. Then $Z(x ; \sigma, \cdot)-Z(x ; s, \cdot)$ and $\int_{0}^{\cdot}\left(\int_{s}^{\sigma} h^{2}(u, v) d u\right)^{1 / 2} d w(v)$ are equivalent processes, where $w$ is a standard Wiener process on $[0, T]$.

We are now ready to establish the following theorem.

Theorem 2. Let $\theta(s, w(\cdot))$ be a bounded function on $[0, S] \times C[0, T]$, and continuous in $(s, w(\cdot))$ in the uniform topology for $w$. Let $\psi \in \mathscr{A}(C[0, T])$, $\lambda>0$, and $\rho=\lambda^{-1 / 2}$. Let $F_{s}(Z(x))$ and $I_{\lambda, Q_{s}}^{h}\left(F_{s}\right) \psi$ be defined by (3.1) and (3.2), respectively. Then, for each $\eta \in C[0, T]$, the function

$$
G(s, \eta(\cdot)) \equiv\left(I_{\lambda, Q_{s}}^{h}\left(F_{s}\right) \psi\right)(\eta(\cdot))
$$

satisfies the Kac-Feynman Wiener integral equation

$$
\begin{aligned}
G(s, \eta(\cdot)) & =E_{w}\left[\psi\left(\rho \int_{0}^{\cdot}\left(\int_{s}^{s} h^{2}(u, v) d u\right)^{1 / 2} d w(v)+\eta(\cdot)\right)\right] \\
& +\int_{s}^{S} E_{w}\left[(\theta \cdot G)\left(\sigma, \rho \int_{0}^{\cdot}\left(\int_{s}^{\sigma} h^{2}(u, v) d u\right)^{1 / 2} d w(v)+\eta(\cdot)\right)\right] d \sigma,
\end{aligned}
$$

where $(\theta \cdot G)(u, v)=\theta(u, v) G(u, v)$.

Proof. Since $F_{s}(Z(x))=\exp \left\{\int_{s}^{S} \theta(\sigma, Z(x ; \sigma, \cdot)) d \sigma\right\}$, it follows that

$$
\frac{d}{d s} F_{s}(\rho Z(x)+\eta)=-\theta(s, \rho Z(x ; s, \cdot)+\eta(\cdot)) F_{s}(\rho Z(x)+\eta) .
$$

Integrate over $[s, S]$ to obtain

$$
1-F_{s}(\rho Z(x)+\eta)=-\int_{s}^{s} \theta(\sigma, \rho Z(x ; \sigma, \cdot)+\eta(\cdot)) F_{\sigma}(\rho Z(x)+\eta) d \sigma .
$$


Multiply each member by $\psi(\rho Z(x ; S, \cdot)+\eta(\cdot))$ and take the conditional expectation with conditioning $Z(x ; s, 0)=0$ to obtain

$$
\begin{aligned}
& E_{x}[\psi(\rho Z(x ; S, \cdot)+\eta(\cdot)) \mid Z(x ; s, \cdot)=0]-G(s, \eta(\cdot)) \\
& =-\int_{s}^{S} E_{x}\left[\theta(\sigma, \rho Z(x ; \sigma, \cdot)+\eta(\cdot)) F_{\sigma}(\rho Z(x)+\eta)\right. \\
& \cdot \psi(\rho Z(x ; S, \cdot)+\eta(\cdot)) \mid Z(x ; s, \cdot)=0] d \sigma .
\end{aligned}
$$

Noting that for $0 \leq s \leq \sigma \leq S, Z(x ; \sigma, \cdot)-Z(x ; s, \cdot)$ and $Z(x ; s, \cdot)$ are independent processes, it follows from (3.5) that

$$
\begin{aligned}
& E_{x}[\psi( \rho \\
&\{Z(x ; S, \cdot)-Z(x ; s, \cdot)\}+\eta(\cdot))]-G(s, \eta(\cdot)) \\
&=-\int_{s}^{S} E_{x}[\theta(\sigma, \rho\{Z(x ; \sigma, \cdot)-Z(x ; s, \cdot)\}+\eta(\cdot)) \\
& \cdot F_{\sigma}(\rho\{F(x ; *, \cdot)-Z(x ; s, \cdot)\}+\eta(\cdot)) \\
&\cdot \psi(\rho\{Z(x ; S, \cdot)-Z(x ; s, \cdot)\}+\eta(\cdot))] d \sigma \\
&=-\int_{s}^{s} E_{x}[\theta(\sigma, \rho\{Z(x ; \sigma, \cdot)-Z(x ; s, \cdot)\}+\eta(\cdot)) \\
& \quad \cdot F_{\sigma}(\rho\{Z(x ; *, \cdot)-Z(x ; \sigma, \cdot)+Z(x ; \sigma, \cdot)-Z(x ; s, \cdot)\}+\eta(\cdot)) \\
&\quad \cdot \psi(\rho\{Z(x ; S, \cdot)-Z(x ; \sigma, \cdot)+Z(x ; \sigma, \cdot)-Z(x ; s, \cdot)\}+\eta(\cdot))] d \sigma .
\end{aligned}
$$

Checking the definition of $F_{s}(Z(x ; *, \cdot))$ given by $(3.1)$, we see that the value of ' *' ranges between $S$ and $S$. Accordingly, the value of $*$ in the expression $F_{\sigma}(\rho Z(x ; *, \cdot)+\eta(\cdot))$ ranges between $\sigma$ and $S$. Thus, $Z(x ; *, \cdot)-Z(x ; \sigma, \cdot)$ and $Z(x ; \sigma, \cdot)-Z(x ; s, \cdot)$ appearing in the last expression of (3.6) are independent. Upon applying Lemma 2, we may rewrite (3.6) in the form

$$
\begin{array}{r}
E_{w}\left[\psi\left(\rho \int_{0}\left(\int_{s}^{S} h^{2}(u, v) d u\right)^{1 / 2} d w(v)+\eta(\cdot)\right)\right]-G(s, \eta(\cdot)) \\
=-\int_{s}^{S} E_{x}\left(E _ { w } \left[\theta\left(\sigma, \rho \int_{0}^{\cdot}\left(\int_{s}^{\sigma} h^{2}(u, v) d u\right)^{1 / 2} d w(v)+\eta(\cdot)\right)\right.\right. \\
\cdot F_{\sigma}(\rho\{Z(x ; *, \cdot)-Z(x ; \sigma, \cdot)\} \\
\left.+\rho \int_{0}^{\cdot}\left(\int_{s}^{\sigma} h^{2}(u, v) d u\right)^{1 / 2} d w(v)+\eta(\cdot)\right) \\
\cdot \psi(\rho\{Z(x ; S, \cdot)-Z(x ; \sigma, \cdot)\} \\
\left.\left.\left.+\rho \int_{0}^{\cdot}\left(\int_{s}^{\sigma} h^{2}(u, v) d u\right)^{1 / 2} d w(v)+\eta(\cdot)\right)\right]\right) d \sigma .
\end{array}
$$


Using the Fubini theorem to switch the order of the inner integrations on the right-hand side of (3.7), and then recognizing the resulting expression as a conditional expectation, we may write (3.7) in the form

$$
\begin{gathered}
E_{w}\left[\psi\left(\rho \int_{0}\left(\int_{s}^{s} h^{2}(u, v) d u\right)^{1 / 2} d w(v)+\eta(\cdot)\right)\right]-G(s, \eta(\cdot)) \\
=-\int_{s}^{S} E_{w}\left\{\theta\left(\sigma, \rho \int_{0}\left(\int_{s}^{\sigma} h^{2}(u, v) d u\right)^{1 / 2} d w(v)+\eta(\cdot)\right)\right. \\
\cdot E_{x}\left[F_{\sigma}\left(\rho Z(x ; *, \cdot)+\rho \int_{0}^{\cdot}\left(\int_{s}^{\sigma} h^{2}(u, v) d u\right)^{1 / 2} d w(v)+\eta(\cdot)\right)\right. \\
\cdot \psi\left(\rho Z(x ; S, \cdot)+\rho \int_{0}^{\cdot}\left(\int_{s}^{\sigma} h^{2}(u, v) d u\right)^{1 / 2} d w(v)+\eta(\cdot)\right) \\
\mid Z(x ; \sigma, \cdot)=0]\} d \sigma .
\end{gathered}
$$

Using (3.2) and (3.3), (3.8) becomes

$$
\begin{aligned}
E_{w}\left[\psi\left(\rho \int_{0}\left(\int_{s}^{s} h^{2}(u, v) d u\right)^{1 / 2} d w(v)+\eta(\cdot)\right)\right]-G(s, \eta(\cdot)) \\
=-\int_{s}^{S} E_{w}\left[\theta\left(\sigma, \rho \int_{0}^{\cdot}\left(\int_{s}^{\sigma} h^{2}(u, v) d u\right)^{1 / 2} d w(v)+\eta(\cdot)\right)\right. \\
\left.\cdot G\left(\sigma, \rho \int_{0}^{\cdot}\left(\int_{s}^{\sigma} h^{2}(u, v) d u\right)^{1 / 2} d w(v)+\eta(\cdot)\right)\right] d \sigma,
\end{aligned}
$$

and hence the proof is complete.

We now give two corollaries to Theorem 2.

Corollary 1. If $h \equiv 1$ on $Q$, then (3.4) becomes

$$
\begin{aligned}
G(s, \eta(\cdot))= & E_{w}[\psi(\rho \sqrt{S-s} w(\cdot)+\eta(\cdot))] \\
& +\int_{s}^{s} E_{w}[(\theta \cdot G)(\sigma, \rho \sqrt{\sigma-s} w(\cdot)+\eta(\cdot))] d \sigma,
\end{aligned}
$$

which agrees with Theorem A given by Cameron and Storvick.

Corollary 2. Let $\phi(\sigma, \tau, y(\cdot))$ be bounded and continuous on $Q \times C[0, T]$ (in the uniform topology for $y)$. Let $\xi(u)$ be defined on $\mathbb{R}$ such that $\xi(u) \exp \left(-\gamma u^{2}\right)$ is integrable on $\mathbb{R}$ for every $\gamma>0$. Let $G$ be given by

$$
\begin{array}{r}
G(s, \eta(\cdot))=E_{x}\left[\exp \left\{\int_{0}^{T} \int_{s}^{S} \phi(\sigma, \tau, \rho Z(x ; \sigma, \tau)+\eta(\tau)) d \sigma d \tau\right\}\right. \\
\cdot \xi(\rho Z(x ; S, T)+\eta(T)) \mid x(s, \cdot)=0] .
\end{array}
$$


Then $G$ satisfies the following integral equation:

$$
\begin{array}{r}
G(s, \eta(\cdot))=E_{w}\left[\xi\left(\rho \int_{0}^{T}\left(\int_{s}^{S} h^{2}(u, v) d u\right)^{1 / 2} d w(v)+\eta(T)\right)\right] \\
+E_{w}\left[\int_{s}^{S} \int_{0}^{T} \phi\left(\sigma, \tau, \rho \int_{0}^{\tau}\left(\int_{s}^{\sigma} h^{2}(u, v) d u\right)^{1 / 2} d w(v)+\eta(\tau)\right) d \tau\right. \\
\left.d \cdot G\left(\sigma, \rho \int_{0}^{\cdot}\left(\int_{s}^{\sigma} h^{2}(u, v) d u\right)^{1 / 2} d w(v)+\eta(\cdot)\right) d \sigma\right] .
\end{array}
$$

Proof. This corollary follows from Theorem 2 by setting $\sigma(s, y)=$ $\int_{0}^{T} \phi(s, v, y(v)) d v$ and $\psi(y(\cdot))=\xi(y(T))$.

\section{SOlUTION OF THE INTEGRAL EQUATION}

In $\S 3$ it was shown that for each $\eta \in C[0, T]$ and $\psi \in \mathscr{A}(C[0, T])$, the function

$$
G(s, \eta(\cdot)) \equiv\left(I_{\lambda, Q_{s}}^{h}\left(F_{s}\right) \psi\right)(\eta(\cdot))
$$

satisfies the Kac-Feynman Wiener integral equation (3.4) under a suitable assumption on $\theta$. We show here that the integral equation has a unique solution.

Our first lemma follows easily by checking the mean and covariance functions.

Lemma 3. Let $k_{1}, k_{2} \in L_{2}[0, T]$, and let $w$ and $w_{1}$ be two independent standard Wiener processes on $[0, T]$. Then $\int_{0}^{*} k_{1}(u) d w(u)+\int_{0}^{\cdot} k_{2}(u) d w_{1}(u)$ and $\int_{0}^{\cdot} \sqrt{k_{1}^{2}(u)+k_{2}^{2}(u)} d w(u)$ are equivalent processes on $[0, T]$.

Lemma 4. Let $h \in L_{2}(Q), \psi \in \mathscr{A}(C[0, T])$, and let

$$
A(s, \eta(\cdot)) \equiv E_{w}\left[\left|\psi\left(\rho \int_{0}^{\cdot}\left(\int_{s}^{S} h^{2}(u, v) d u\right)^{1 / 2} d w(v)+\eta(\cdot)\right)\right|\right]
$$

for $\eta \in C[0, T]$. Then

$$
E_{w}\left[A\left(\sigma, \rho \int_{0}^{\cdot}\left(\int_{s}^{S} h^{2}(u, v) d u\right)^{1 / 2} d w(v)+\eta(\cdot)\right)\right]=A(s, \eta(\cdot)) .
$$

Proof. Using the definition of $A(\cdot, \cdot)$, we may write the left-hand side of $(4.2)$ as

$$
\begin{aligned}
I \equiv E_{w}\left(E _ { w _ { 1 } } \left[\mid \psi\left(\rho \int_{0}^{\cdot}\left(\int_{\sigma}^{S} h^{2}(u, v) d u\right)^{1 / 2} d w_{1}(v)\right.\right.\right. \\
\left.\left.\left.+\rho \int_{0}^{\cdot}\left(\int_{s}^{\sigma} h^{2}(u, v) d u\right)^{1 / 2} d w(v)+\eta(\cdot)\right) \mid\right]\right) .
\end{aligned}
$$

An application of Lemma 3 to the last expression yields

$$
I \equiv E_{w}\left[\left|\psi\left(\rho \int_{0}\left(\int_{s}^{S} h^{2}(u, v) d u\right)^{1 / 2} d w(v)+\eta(\cdot)\right)\right|\right],
$$

which completes the proof of the lemma. 
Theorem 3. Let $\theta(s, y(\cdot))$ be bounded by $M$ on $[0, S] \times C[0, T]$ and be continuous there (in the uniform topology for $y$ ), let $\psi \in \mathscr{A}(C[0, T])$, and let $\lambda>0$ with $\rho=\lambda^{-1 / 2}$. Then for $\eta \in C[0, T]$, the solution of the Kac-Feynman Wiener integral equation (3.4) is given by

$$
G(s, \eta(\cdot))=\sum_{j=0}^{\infty} H_{j}(s, \eta(\cdot)),
$$

where the sequence $\left\{H_{j}\right\}$ is given inductively by

$$
H_{0}(s, \eta(\cdot))=E_{w}\left[\psi\left(\rho \int_{0}\left(\int_{s}^{s} h^{2}(u, v) d u\right)^{1 / 2} d w(v)+\eta(\cdot)\right)\right],
$$

and

$$
\begin{aligned}
H_{j+1} & (s, \eta(\cdot)) \\
\quad= & \int_{s}^{s} E_{w}\left[\left(\theta \cdot H_{j}\right)\left(\sigma, \rho \int_{0}\left(\int_{s}^{\sigma} h^{2}(u, v) d u\right)^{1 / 2} d w(v)+\eta(\cdot)\right)\right] d \sigma .
\end{aligned}
$$

The solution $G(s, \eta(\cdot))$ satisfies the inequality

$$
|G(s, \eta(\cdot))| \leq A(s, \eta(\cdot)) \exp \{M S\},
$$

and (4.3) is the only solution satisfying (4.6).

Proof. First, we establish that

$$
\begin{aligned}
\left|H_{j}(s, \eta(\cdot))\right| & \leq \frac{[M(S-s)]^{j}}{j !} A(s, \eta(\cdot)) \\
& \leq \frac{(M S)^{j}}{j !} A(s, \eta(\cdot)) \quad \text { for } j=0,1, \ldots
\end{aligned}
$$

The case $j=0$ follows from (4.1). Assume (4.7) holds for some $j$. Then from (4.5) it follows that

$$
\begin{array}{r}
\left|H_{j+1}(s, \eta(\cdot))\right| \leq \frac{M^{j+1}}{j !} \int_{s}^{S}(\sigma-s)^{j} E_{w}\left[A \left(\sigma, \rho \int_{0}^{\cdot}\left(\int_{s}^{\sigma} h^{2}(u, v) d u\right)^{1 / 2}\right.\right. \\
\cdot d w(v)+\eta(\cdot))] d \sigma .
\end{array}
$$

Next applying (4.2), we obtain

$$
\left|H_{j+1}(s, \eta(\cdot))\right| \leq \frac{M^{j+1}}{j !} A(s, \eta(\cdot)) \int_{s}^{S}(\sigma-s)^{j} d \sigma=\frac{M^{j+1}(S-s)^{j+1}}{(j+1) !} A(s, \eta(\cdot)) .
$$

Thus,

$$
\sum_{j=0}^{\infty}\left|H_{j}(s, \eta(\cdot))\right| \leq A(s, \eta(\cdot)) \exp \{M S\}
$$


and hence, by the dominated convergence theorem,

$$
\begin{gathered}
\int_{s}^{S} E_{w}\left[\theta\left(\sigma, \rho \int_{0}^{\cdot}\left(\int_{s}^{\sigma} h^{2}(u, v) d u\right)^{1 / 2} d w(v)+\eta(\cdot)\right)\right. \\
\left.\cdot \sum_{j=0}^{\infty} H_{j}\left(\sigma, \rho \int_{0}^{\cdot}\left(\int_{s}^{\sigma} h^{2}(u, v) d u\right)^{1 / 2} d w(v)+\eta(\cdot)\right)\right] d \sigma \\
=\sum_{j=0}^{\infty} \int_{s}^{S} E_{w}\left[\theta\left(\sigma, \rho \int_{0}^{\cdot}\left(\int_{s}^{\sigma} h^{2}(u, v) d u\right)^{1 / 2} d w(v)+\eta(\cdot)\right)\right. \\
\left.\cdot H_{j}\left(\sigma, \rho \int_{0}^{\cdot}\left(\int_{s}^{\sigma} h^{2}(u, v) d u\right)^{1 / 2} d w(v)+\eta(\cdot)\right)\right] d \sigma \\
=\sum_{j=0}^{\infty} H_{j+1}(s, \eta(\cdot))=\sum_{j=0}^{\infty} H_{j}(s, \eta(\cdot))-H_{0}(s, \eta(\cdot)) .
\end{gathered}
$$

Therefore,

$$
G(s, \eta(\cdot)) \equiv \sum_{j=0}^{\infty} H_{j}(s, \eta(\cdot))
$$

is a solution of (3.4). Furthermore, using (4.8), $\left.G\left(s, \eta^{\prime} \cdot\right)\right)$ satisfies the inequality (4.6).

To show that $(4.3)$ is the only such solution, assume that $G_{1}(s, \eta(\cdot))$ is another such solution. Then $H \equiv G-G_{1}$ is a solution of the equation

$$
H(s, \eta(\cdot))=\int_{s}^{S} E_{w}\left[( \theta \cdot H ) \left(\sigma, \rho \int_{0}^{\cdot}\left(\int_{s}^{\sigma} h^{2}(u, v) d u\right)^{1 / 2}\right.\right.
$$

$$
\cdot d w(v)+\eta(\cdot))] d \sigma
$$

satisfying the condition

$$
|H(s, \eta(\cdot))| \leq 2 A(s, \eta(\cdot)) \exp \{M S\} .
$$

The use of (4.10) in (4.9) together with the fact that $|\theta(\cdot, \cdot)| \leq M$ yields

$$
\begin{aligned}
|H(s, \eta(\cdot))| & \leq 2 M \exp \{M S\} \int_{s}^{S} E_{w}\left[A \left(\sigma, \rho \int_{0}^{\cdot}\left(\int_{s}^{\sigma} h^{2}(u, v) d u\right)^{1 / 2}\right.\right. \\
& =2 M \exp \{M S\} A(s, \eta(\cdot))(S-s),
\end{aligned}
$$

where the last equality follows from (4.2). Next we use (4.11) in (4.9) and obtain that

$$
|H(s, \eta(\cdot))| \leq 2 M^{2} \exp \{M S\} A(s, \eta(\cdot)) \frac{(S-s)^{2}}{2} .
$$

Repeating this procedure, it follows by induction that

$$
|H(s, \eta(\cdot))| \leq \exp \{M S\} A(s, \eta(\cdot)) \frac{[M(S-s)]^{n}}{n !}, \quad n=1,2,3, \ldots
$$


Thus $H(s, \eta(\cdot))=0$, and hence the uniqueness of the solution is established.

The following corollary to Theorem 3 follows from the fact that

$$
\begin{aligned}
\left(I_{\lambda, Q_{s}}^{h}\left(F_{s}\right) \psi\right)(\eta(\cdot))= & E_{x}\left[\exp \left\{\int_{s}^{S} \theta(\sigma, \rho Z(x ; \sigma, \cdot)+\eta(\cdot)) d \sigma\right\}\right. \\
& \cdot \psi(\rho Z(x ; S, \cdot)+\eta(\cdot)) \mid Z(x ; s, \cdot)=0] \\
= & G(s, \eta(\cdot)) .
\end{aligned}
$$

Corollary 3. Under the same hypotheses as in Theorem 3,

$$
\begin{aligned}
E_{x}[\exp & \left\{\int_{s}^{S} \theta(\sigma, \rho Z(x ; \sigma, \cdot)+\eta(\cdot)) d \sigma\right\} \\
\cdot & \psi(\rho Z(x ; S, \cdot)+\eta(\cdot)) \mid Z(x ; s, \cdot)=0] \\
= & \sum_{n=0}^{\infty} H_{n}(s, \eta(\cdot)),
\end{aligned}
$$

where the sequence $\left\{H_{n}\right\}$ is the same as in Theorem 3. In particular, setting $s=0$ we have

$$
\begin{array}{r}
E_{x}\left[\exp \left\{\int_{0}^{S} \theta(\sigma, \rho Z(x ; \sigma, \cdot)+\eta(\cdot)) d \sigma\right\} \psi(\rho Z(x ; S, \cdot)+\eta(\cdot))\right] \\
=E_{w}\left[\psi\left(\rho \int_{0}\left(\int_{0}^{S} h^{2}(u, v) d u\right)^{1 / 2} d w(v)+\eta(\cdot)\right)\right] \\
+\sum_{n=1}^{\infty} \int_{\Delta_{n}(S)} E_{w_{1}, \ldots, w_{n+1}}\left\{\left[\prod _ { j = 1 } ^ { n } \theta \left(\sigma_{j}, \rho \sum_{k=1}^{j} \int_{0}^{\cdot}\left(\int_{\sigma_{k-1}}^{\sigma_{k}} h^{2}(u, v) d u\right)^{1 / 2}\right.\right.\right. \\
\left.\left.\cdot d w_{k}(v)+\eta(\cdot)\right)\right] \\
\left.\cdot \psi\left(\rho \sum_{k=1}^{n+1} \int_{0}^{\cdot}\left(\int_{\sigma_{k-1}}^{\sigma_{k}} h^{2}(u, v) d u\right)^{1 / 2} d w_{k}(v)+\eta(\cdot)\right)\right\} d \vec{\sigma}
\end{array}
$$

where

$$
\Delta_{n}(S)=\left\{\vec{\sigma}=\left(\sigma_{1}, \ldots, \sigma_{n}\right): 0=\sigma_{0}<\sigma_{1}<\cdots<\sigma_{n}<\sigma_{n+1}=S\right\} .
$$

Example 3. Using Corollary 3, we can express the Yeh-Wiener integral

$$
I \equiv E_{x}\left[\exp \left\{\int_{0}^{S} \int_{0}^{T} \phi\left(\sigma, \tau, \int_{0}^{\tau} \int_{0}^{\sigma} h(u, v) d x(u, v)\right) d \tau d \sigma\right\}\right]
$$

as a sum of Lebesgue integrals, where $\phi(s, t, u)$ is a bounded continuous function on $Q \times \mathbb{R}$. To do so we choose $\psi \equiv 1, \rho=1, \eta \equiv 0$ and we let

$$
\theta(s, y(\cdot))=\int_{0}^{T} \phi(s, \tau, y(\tau)) d \tau
$$


In addition for $n=1,2, \ldots$ let

$$
\Delta_{n}(S)=\left\{\vec{\sigma}=\left(\sigma_{1}, \ldots, \sigma_{n}\right): 0=\sigma_{0}<\sigma_{1}<\cdots<\sigma_{n}<S\right\}
$$

Then using (4.13) we have

$$
\begin{aligned}
I \equiv & E_{x}\left[\exp \left\{\int_{0}^{S} \phi\left(\sigma, \int_{0} \int_{0}^{\sigma} h(u, v) d x(u, v)\right) d \sigma\right\}\right] \\
=1+\sum_{n=1}^{\infty} \int_{\Delta_{n}(S)} E_{w_{1}, \ldots, w_{n}} & \cdot\left\{\prod_{j=1}^{n}\left[\theta\left(\sigma_{j}, \sum_{k=1}^{j} \int_{0}^{\cdot}\left(\int_{\sigma_{k-1}}^{\sigma_{k}} h^{2}(u, v) d u\right)^{1 / 2} d w_{k}(v)\right)\right]\right\} d \vec{\sigma} \\
=1 & +\sum_{n=1}^{\infty} \int_{\Delta_{n}(S)} E_{w_{1}, \ldots, w_{n}} \\
& \cdot\left\{\prod_{j=1}^{n}\left[\int_{0}^{T} \phi\left(\sigma_{j}, \tau_{j}, \sum_{k=1}^{j} \int_{0}^{\tau_{j}}\left(\int_{\sigma_{k-1}}^{\sigma_{k}} h^{2}(u, v) d u\right)^{1 / 2} d w_{k}(v)\right) d \tau_{j}\right]\right\} d \vec{\sigma} \\
= & 1+\sum_{n=1}^{\infty} \int_{\Delta_{n}(S)} \int_{[0, T]^{n}} E_{w_{1}, \ldots, w_{n}} \\
& \cdot\left\{\prod_{j=1}^{n} \phi\left(\sigma_{j}, \tau_{j}, \sum_{k=1}^{j} \int_{0}^{\tau_{j}}\left(\int_{\sigma_{k-1}}^{\sigma_{k}} h^{2}(u, v) d u\right)^{1 / 2} d w_{k}(v)\right)\right\} d \vec{\tau} d \vec{\sigma}
\end{aligned}
$$

To evaluate the Wiener integrals involved above, we introduce the following notation: For $1 \leq j \leq n$, define

$$
\begin{aligned}
& W_{n-j+1}\left(u_{j, j}, u_{j, j+1}, \ldots, u_{j, n} ; \tau_{j}, \ldots, \tau_{n}\right) \\
& \quad=\left\{\begin{array}{l}
0 \text { if } \tau_{1}, \ldots, \tau_{n} \text { are not all distinct, } \\
\prod_{k=j}^{n}\left[2 \pi \int_{\tau_{\alpha_{k-1}}}^{\tau_{\alpha_{k}}} h^{2}\right]^{-1 / 2} \exp \left[-\frac{\left(u_{j, \alpha_{k}}-u_{j, \alpha_{k-1}}\right)^{2}}{\left.2 \int_{\tau_{\alpha_{k-1}}}^{\tau_{\alpha_{k}} \int_{\sigma_{j-1}}^{\sigma_{j}} h^{2}}\right] \text { otherwise },}\right.
\end{array}\right.
\end{aligned}
$$

where $\left(\alpha_{j}, \ldots, \alpha_{n}\right)$ is a permutation of $(j, \ldots, n)$ such that $\tau_{\alpha_{j}}<\tau_{\alpha_{j+1}}<$ $\cdots<\tau_{\alpha_{n}}$ and $\alpha_{j-1}=0, \tau_{0}=0$, and $u_{j, 0}=0$. Then, we have

$$
\begin{array}{r}
I=1+\sum_{n=1}^{\infty} \int_{\Delta_{n}(S)} \int_{[0, T]^{n}} \int_{\mathbf{R}^{n(n+1) / 2}} \prod_{j=1}^{n}\left\{W_{n-j+1}\left(u_{j, j}, \ldots, u_{j, n} ; \tau_{j}, \ldots, \tau_{n}\right)\right. \\
\left.\cdot \phi\left(\sigma_{j}, \tau_{j}, \sum_{k=1}^{j} u_{k, j}\right)\right\} d \vec{u} d \vec{\tau} d \vec{\sigma}
\end{array}
$$


Example 4. Let $\phi$ and $\theta$ be as in Example 3 above. Then

$$
\begin{aligned}
& E_{x}\left[\int_{0}^{S} \int_{0}^{T} \phi\left(\sigma, \tau, \int_{0}^{\tau} \int_{0}^{\sigma} h(u, v) d x(u, v)\right) d \tau d \sigma\right] \\
& \quad=\int_{0}^{S} \int_{0}^{T}\left[2 \pi \int_{0}^{\tau} \int_{0}^{\sigma} h^{2}\right]^{-1 / 2} \int_{\mathbb{R}} \phi(\sigma, \tau, u) \exp \left\{-\frac{u^{2}}{2 \int_{0}^{\tau} \int_{0}^{\sigma} h^{2}}\right\} d u d \tau d \sigma \\
& \quad=\int_{0}^{S} E_{w}\left[\theta\left(\sigma, \int_{0}^{\cdot}\left(\int_{0}^{\sigma} h^{2}(u, v) d u\right)^{1 / 2} d w(v)\right)\right] d \sigma .
\end{aligned}
$$

\section{REFERENCES}

1. R. H. Cameron and D. A. Storvick, An operator valued function space integral and a related integral equation, J. Math. Mech. 18 (1968), 517-552.

2. __, An integral equation related to the Schroedinger equation with an application to integration in function space, Problems in Analysis, Princeton Univ. Press, Princeton, NJ, 1970, pp. 175-193.

3. _ An operator valued function space integral applied to functions of class $L_{2}$, J. Math. Anal. Appl. 42 (1973), 330-373.

4. _ An operator valued Yeh-Wiener integral, and a Wiener integral equation, Indiana Univ. Math. J. 25 (1976), 235-258.

5. D. M. Chung, C. Park, and D. L. Skoug, Operator-valued Feynman integrals via conditional Feynman integrals, Pacific J. Math. 146 (1990), 21-42.

6. J. L. Doob, Stochastic processes, Wiley, New York, 1965.

7. T. Hida, Brownian motion, Springer-Verlag, Berlin and New York, 1980.

8. G. W. Johnson and D. L. Skoug, A Banach algebra of Feynman integrable functionals with application to an integral equation formally equivalent to Schroedinger's equation, J. Funct. Anal. 12 (1973), 129-152.

9. C. Park, On Fredholm transformations in Yeh-Wiener space, Pacific J. Math. 40 (1972), 173-195.

10. C. Park and D. L. Skoug, $A$ simple formula for conditional Wiener integrals with application, Pacific J. Math. 135 (1988), 381-394.

11. _ Conditional Yeh-Wiener integrals with vector-valued conditioning functions, Proc. Amer. Math. Soc. 105 (1989), 450-461.

12. J. Yeh, Wiener measure in a space of functions of two variables, Trans. Amer. Math. Soc. 95 (i 960$), 433-450$.

13. __ Stochastic processes and the Wiener integral, Marcel Dekker, New York, 1983.

Department of Mathematics and Statistics, Miami University, Oxford, Ohio 45056

E-mail address: cpark@miavx1.acs.muohio.edu

Department of Mathematics and Statistics, University of Nebraska, Lincoln, NeBRASKA 68588-0323

E-mail address: dskoug@hoss.unl.edu 